\title{
Two Molecular Clouds with Anomalous Velocities in The Galactic Anticenter
}

\author{
Youngung Lee ${ }^{1}$, Young Sik Kim ${ }^{1,2}$, Hyung-Goo Kim ${ }^{1}$, JAe-Hoon Jung ${ }^{1}$, In-Sung Yim ${ }^{1}$, Hyunwoo Kang ${ }^{1}$, \\ Changhoon Lee ${ }^{1}$, Bong-Gyu Kim ${ }^{1}$, and Kwang-Tae Kim ${ }^{2}$ \\ ${ }^{1}$ Korea Astronomy and Space Science Institute, 776 Daedukdae-ro, Yuseong, Daejeon 305-348, Korea; yulee@kasi.re.kr \\ ${ }^{2}$ Department of Space and Astronomy, Chungnam National University, Daejeon 305-764, Korea
}

Received November 14, 2014; accepted December 8, 2014

\begin{abstract}
We map two molecular clouds located in the exact anticenter region emitting in the $(J=1-0)$ transition of ${ }^{12} \mathrm{CO}$ and ${ }^{13} \mathrm{CO}$ using the 3-mm SIS mixer receiver on the 14 -m radio telescope at Taeduk Radio Astronomy Observatory. The target clouds with anomalous velocities of $V_{\mathrm{LSR}} \sim-20 \mathrm{~km} \mathrm{~s}^{-1}$ are distinguished from other clouds in this direction. In addition, they are located in the interarm region between the Orion Arm and the Perseus Arm. Sizes of the clouds are estimated to be about 8.6 and $10.8 \mathrm{pc}$, respectively. The total mass is estimated to be about $4 \times 10^{3} \mathrm{M}_{\odot}$ using CO luminosity of the clouds. Several cores are detected, but no sign of star formation is found according to the IRAS point sources. Their larger linewidths, anomalous velocities, and their location at the interarm region make these clouds more distinguished, though their physical properties are similar to the dark clouds in the solar neighborhood in terms of mass and size.
\end{abstract}

Key words: Galaxy: anticenter, local interstellar matter - ISM: molecules, kinematics and dynamics radio lines: ISM

\section{INTRODUCTION}

The Galactic anticenter region of $(l, b)=\left(178 .^{\circ} 0-\right.$ $\left.186^{\circ} .0,3 .^{\circ} 5-6 .^{\circ} 0\right)$ was previously mapped on a $3^{\prime}$ grid in the transition of ${ }^{12} \mathrm{CO}(J=1-0)$ line using the 14-m radio telescope at Taeduk Radio Astronomy Observatory (Lee et al. 1999; Paper I). They identified two molecular clouds with anomalous velocities of about $V_{\mathrm{LSR}}=-20 \mathrm{~km} \mathrm{~s}^{-1}$. Toward the exact Galactic anticenter region $\left(l=180^{\circ}\right)$, the LSR velocity is presumably expected to be around $V_{\mathrm{LSR}}=0 \mathrm{~km} \mathrm{~s}^{-1}$. The velocity of $V_{\mathrm{LSR}}=-20 \mathrm{~km} \mathrm{~s}^{-1}$ seems to be quite peculiar for the cloud in this exact anticenter region, even considering a possible streaming motion of $7 \mathrm{~km} \mathrm{~s}^{-1}$ (Blitz 1991) in the spiral arm. This motivated us to undertake more detailed study on the physical conditions for these molecular clouds with peculiar velocity.

In order to study the physical properties of a molecular cloud, distance information is essential. In fact, Kim et al. (2000) conducted deep CCD observations in $\mathrm{V}$ band to obtain stellar density distribution and to determine the distances to these two molecular clouds. Star count method based on the linear programming technique was applied to the CCD photometric data. They found that the two molecular clouds are located almost at the same distance of about $1.1 \pm 0.1 \mathrm{kpc}$, and the peak extinction caused by the clouds was estimated to be $\sim 2.2 \pm 0.3 \mathrm{mag}$ in $\mathrm{V}$ band.

In this paper we have mapped these clouds with a higher sampling rate of $1^{\prime}$ for further study of the distribution and physical condition of molecular gas. Observational mode is described in Section 2. We present

Corresponding AUthor: Y. Lee our results along with physical properties of the clouds in Section 3. In Section 4 we delineate the some highlights of the observational results as well as discussion. We summarize our results in the final section.

\section{Observations}

We have mapped about $1 \mathrm{deg}^{2}$ section of $(l, b)=$ $\left(179^{\circ} .8-181^{\circ} .0,5^{\circ} .0-6^{\circ} .0\right)$, where the clouds with anomalous velocity are residing, in the $(J=1-0)$ transitions of ${ }^{12} \mathrm{CO}$ and ${ }^{13} \mathrm{CO}$ using the 3 -mm SIS mixer receiver on the Taeduk Radio Astronomy Observatory (TRAO) 14-m radio telescope. The beam size (FWHM) is about $50^{\prime \prime}$ and the grid spacing of the new map is $1^{\prime}$. We used a $250 \mathrm{KHz}$ filterbank, which covers a velocity range of $170 \mathrm{~km} \mathrm{~s}^{-1}$ with a resolution of $0.65 \mathrm{~km} \mathrm{~s}^{-1}$ at ${ }^{12} \mathrm{CO}(J=1-0)$. The filterbanks were centered at $V_{\mathrm{LSR}}=0 \mathrm{~km} \mathrm{~s}^{-1}$ for all observations. Scale of the target region is about 2 deg by 1.5 degree, and only the clouds with anomalous velocity were observed on $1^{\prime}$ grid. The rest of the data was taken from the previous observations onto $3^{\prime}$-grid (Paper I).

All observations were conducted by position switching between mapping positions and reference positions which were carefully selected to be free of $\mathrm{CO}$ emission. Each reference observation was shared with observations at 2 to 4 mapping positions depending on the sky stability. Calibration was accomplished by frequently observing an ambient temperature load. All antenna temperatures quoted are corrected for atmospheric extinction and for the forward spillover and scattering losses of the antenna and radome $\left(\eta_{f s s}=0.63\right.$ at 115 $\mathrm{GHz}$ ), and are therefore on the temperature scale defined by Kutner \& Ulich (1981). The system tempera- 


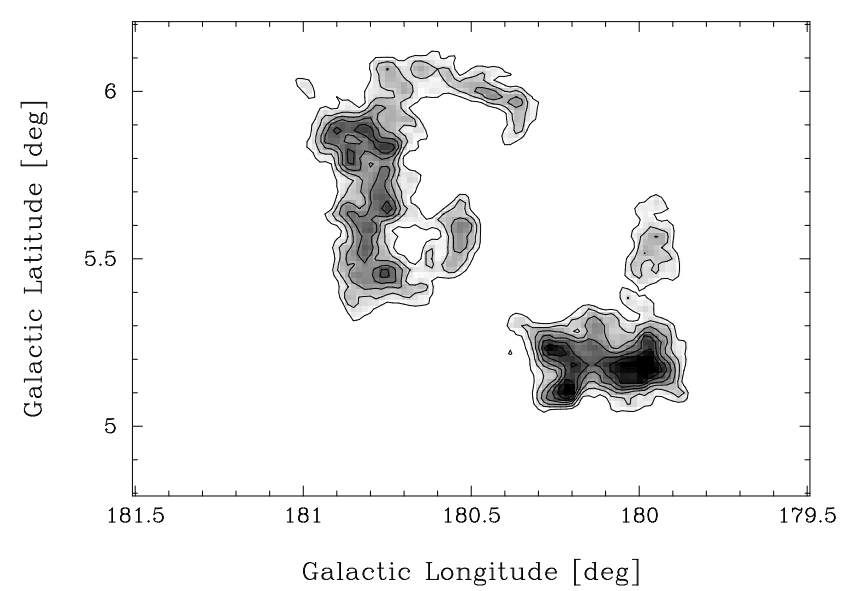

Figure 1. The ${ }^{12} \mathrm{CO}$ integrated intensity map of anomalous velcity range of $V_{\mathrm{LSR}}=-30$ to $-10 \mathrm{~km} \mathrm{~s}^{-1}$. The first contour and its increment are $2 \mathrm{~K} \mathrm{~km} \mathrm{~s}^{-1}$. Grey scale ranges from 2 to $14 \mathrm{~K} \mathrm{~km} \mathrm{~s}^{-1}$. Cloud $\mathrm{A}$ is located at righthand side, and Cloud $\mathrm{B}$ is at lefthand side.

ture corrected for the atmosphere varied typically from 400 to $600 \mathrm{~K}$ at ${ }^{13} \mathrm{CO}(J=1-0)$, and from 700 to 900 $\mathrm{K}$ at ${ }^{12} \mathrm{CO}(J=1-0)$ depending on the elevation and the weather condition.

All spectra were examined visually both before and after fitting the baseline, linear or polynomial. Some of spectra were reobserved and replaced when their quality was significantly low. The collected spectra were transformed into IRAF data format using modified FCRAO task package, which was developed at Five College Radio Astronomy Observatory. Most of the subsequent analysis was done within IRAF using this package. The average rms noise of the data is estimated to be $\sigma_{r m s} \sim$ $0.2 \mathrm{~K}$ for ${ }^{12} \mathrm{CO}(J=1-0)$ in $T_{\mathrm{R}}^{*}$ at a velocity resolution of $0.65 \mathrm{~km} \mathrm{~s}^{-1}$, and $\sigma_{r m s} \sim 0.1 \mathrm{~K}$ for ${ }^{13} \mathrm{CO}(J=1-0)$ in $T_{\mathrm{R}}^{*}$ at a velocity resolution of $0.68 \mathrm{~km} \mathrm{~s}^{-1}$.

\section{Results}

\subsection{CO Emission}

A total number of about 2,200 spectra was obtained in the ${ }^{12} \mathrm{CO}(J=1-0)$, and about 900 spectra were obtained in the ${ }^{13} \mathrm{CO}(J=1-0)$ for the strong ${ }^{12} \mathrm{CO}$ emission region. We resampled the previously observed region with $3^{\prime}$-grid spacing onto $1^{\prime}$-grid spacing; thus, the map other than newly observed region was substantially undersampled. However, we consider the rest part other than the target clouds could be ignored in this study, as we are only interested in those two molecular clouds with peculiar velocity. The mapped region covers the entire extent of the two clouds with anomalous velocities in the ${ }^{12} \mathrm{CO}(J=1-0)$. The ${ }^{13} \mathrm{CO}$ ( $J=1-0)$ observations were focused only on the strong emission region of ${ }^{12} \mathrm{CO}(J=1-0)$, as only brighter ${ }^{12} \mathrm{CO}(J=1-0)$ emission region can produce the ${ }^{13} \mathrm{CO}$ emission enough to to detected.

The spectral intensity maps integrated from $V_{\mathrm{LSR}}=$ -25 to $-15 \mathrm{~km} \mathrm{~s}^{-1}$ are presented in Figures 1 and 2 . Two clouds have clear boundaries and are composed of

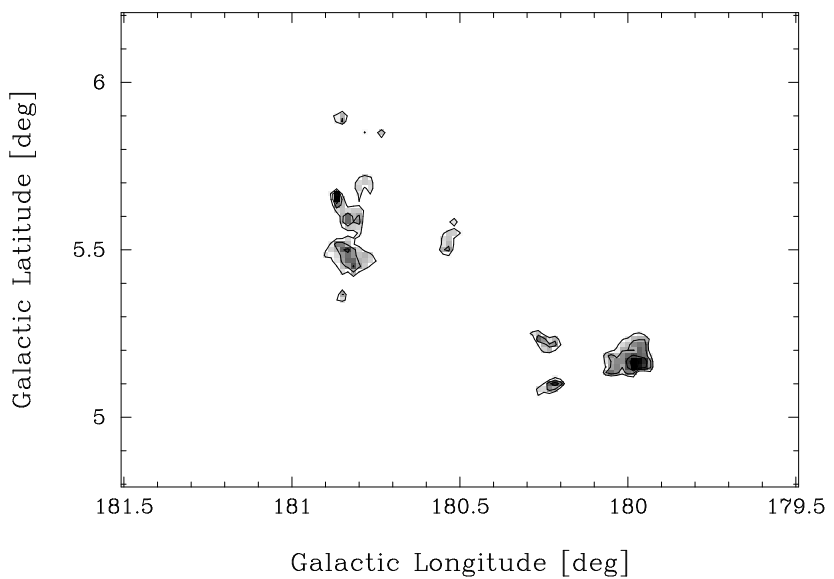

Figure 2. The ${ }^{13} \mathrm{CO}$ integrated intensity map of anomalous velcity range of $V_{\mathrm{LSR}}=-30$ to $-10 \mathrm{~km} \mathrm{~s}^{-1}$. The first contour is $1 \mathrm{~K} \mathrm{~km} \mathrm{~s}^{-1}$ and its increment is $0.5 \mathrm{~K} \mathrm{~km} \mathrm{~s}^{-1}$. Grey scale ranges from 1 to $2.5 \mathrm{~K} \mathrm{~km} \mathrm{~s}^{-1}$.

several clumps and some elongated structures. These two clouds can be handled separately, as Cloud A and Cloud B; Cloud A is in the right side of the mapped region centered on $(l, b)=\left(180^{\circ}, 5^{\circ} .2\right)$, and Cloud $\mathrm{B}$ is in the left side centered on $\left(180^{\circ} .8,5^{\circ} .6\right)$, respectivley.

Another set of ${ }^{12} \mathrm{CO}(J=1-0)$ intensity maps integrated from -10 to $0 \mathrm{~km} \mathrm{~s}^{-1}$, and 0 to +10 $\mathrm{km} \mathrm{s}^{-1}$ are presented in Figures 3 and 4, respectively. The morphologies of the clouds are somewhat clumpy and very similar to dark clouds in the solar neighborhood. One clump is associated with IRAS point source $06048+3123$ at $(l, b)=\left(180^{\circ} .31,5^{\circ} .42\right)$. A probable young stellar object is residing at the center, which is associated with a dense core and IRAS point source $06048+3123$. A single-point observation was conducted by Wouterloot et al. (1989/2000) toward this point source IRAS $06048+3123$. They found that $V_{\mathrm{LSR}}=$ $-5.24 \mathrm{~km} \mathrm{~s}^{-1}$, and ${ }^{12} \mathrm{CO}(J=1-0)$ peak temperature of $10.8 \mathrm{~K}$ in the unit of $T_{\mathrm{R}}^{*}$. FIR luminosity of the IRAS point source is hard to be estimated as its distance is not known. From 2MASS data around this IRAS point source, there are many embedded stars which are reddened at least 2 magnitude in $\mathrm{K}$ band. From the difference in $V_{\mathrm{LSR}}$, it is clear that this point source is not related to our target objects with anomalous velocities.

Position-velocity maps integrated along longitude and latitude are shown in Figures 5 and 6 . Two maps show three discrete velocity ranges of the molecular gas; -25 to $-15 \mathrm{~km} \mathrm{~s}^{-1},-10$ to $0 \mathrm{~km} \mathrm{~s}^{-1}$, and 0 to +10 $\mathrm{km} \mathrm{s}^{-1}$. The last two are within the range of possible streaming motion (Blitz 1991), and some of them are thought to be local. On the other hand, gas components with the velocity range of -25 to $-15 \mathrm{~km} \mathrm{~s}^{-1}$ seems to be very anomalous in this direction. We will discuss this issue in the next section.

\subsection{Velocity Field}

Channel maps of the cloud at individual velocities are shown in Figure 7. For each channel map, two velocity channels of spectrometer were binned into one 


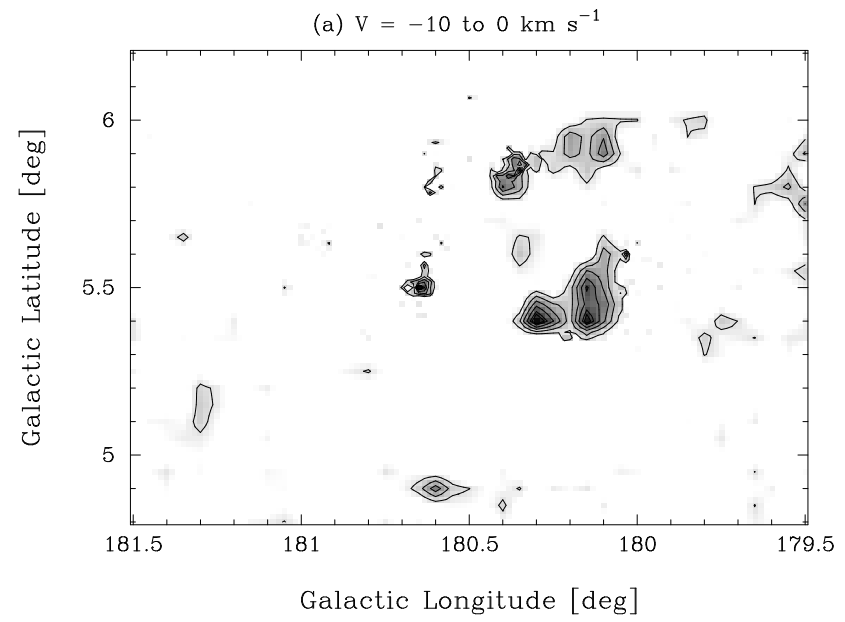

Figure 3. The ${ }^{12} \mathrm{CO}$ integrated intensity map of $V_{\mathrm{LSR}}=-10$ to $0 \mathrm{~km} \mathrm{~s}^{-1}$. The first contour and its increment are $2 \mathrm{~K}$ $\mathrm{km} \mathrm{s}^{-1}$. Grey scale ranges from 2 to $14 \mathrm{~K} \mathrm{~km} \mathrm{~s}^{-1}$.

(b) $\mathrm{V}=0$ to $10 \mathrm{~km} \mathrm{~s}^{-1}$

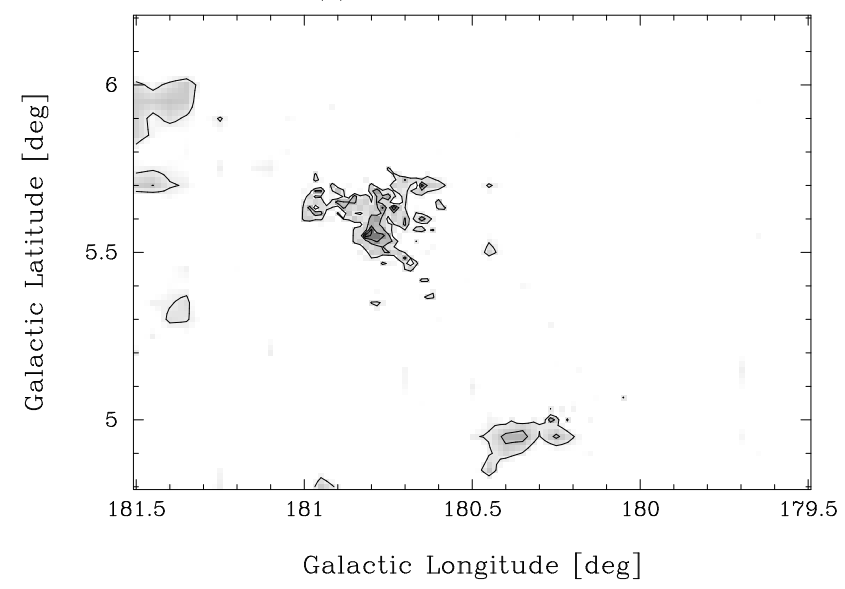

Figure 4. The ${ }^{12} \mathrm{CO}$ integrated intensity map of $V_{\mathrm{LSR}}=0$ to $10 \mathrm{~km} \mathrm{~s}^{-1}$. The first contour and its increment are $2 \mathrm{~K}$ $\mathrm{km} \mathrm{s}^{-1}$. Grey scale ranges from 2 to $14 \mathrm{~K} \mathrm{~km} \mathrm{~s}^{-1}$.

panel (thus, $1.3 \mathrm{~km} \mathrm{~s}^{-1}$ per panel) and the velocity range covered is -27.0 to $-12.7 \mathrm{~km} \mathrm{~s}^{-1}$. These maps clearly show the filamentary structure and partial clumpiness of the molecular gas. We intentionally present the channel map without any smoothing to show the cloud structure more realistically. However, the channel shows no specific dynamic status of the gas within. In fact, the velocity field itself is very similar to other dark clouds in the solar neighborhood, though they have broader linewiths(see next section).

Figures 8 and 9 represents ${ }^{12} \mathrm{CO}(J=1-0)$ linewidth and mean velocity maps of the target cloud, respectively. The mean velocity of the lower part (Cloud A) is around $-20.5 \mathrm{~km} \mathrm{~s}^{-1}$ and that of the upper part (Cloud B) is $-20.0 \mathrm{~km} \mathrm{~s}^{-1}$. For the mean velocity and linewidth maps, we used a threshold-cutoff only for the meaningful region larger than the $3 \sigma$ of the ${ }^{12} \mathrm{CO}$ $(J=1-0)$ integrated intensity of the cloud.

The mean velocity map shows that the velocity at the position $(l, b)=\left(180^{\circ} .8,5^{\circ} .85\right)$ is quite different

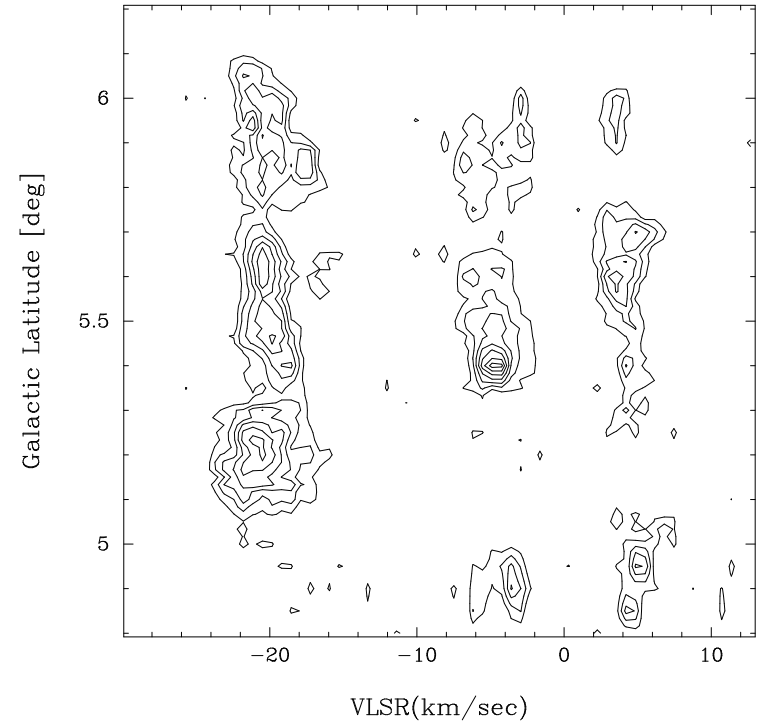

Figure 5. Galactic latitude-velocity integrated intensity map of the target region. The lowest contour level is $14 \mathrm{~K}$ arcmin and the increment between levels is $10 \mathrm{~K}$ arcmin.

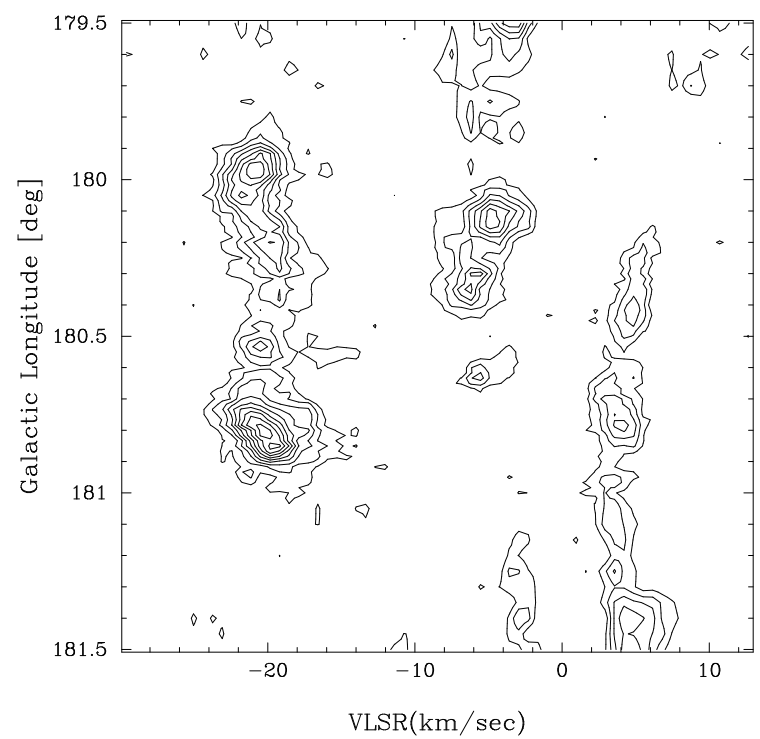

Figure 6. Galactic longitude-velocity integrated intensity map of the target region. The lowest contour level and the increment between levels are $8 \mathrm{~K}$ arcmin.

from those of other parts in Cloud A and B; the mean velocity of the former is more redshifted than that of the remaining part. It is noticeable that the linewidths at that postion is significantly broader than those of the rest of Cloud B.

\subsection{Physical Parameters}

To derive physical parameters of a cloud, we firstly need to estimate linewidths and luminosities along with distance information. Distance to this cloud was established by Kim et al. (2000) as $1.1 \mathrm{kpc}$. With this distance, the cloud area is estimated to be about $1.1 \mathrm{pc}^{2}$ above a $3 \sigma$ rms level of ${ }^{12} \mathrm{CO}(J=1-0)$ integrated intensity. We estimated the ${ }^{12} \mathrm{CO}(J=1-0)$ luminos- 


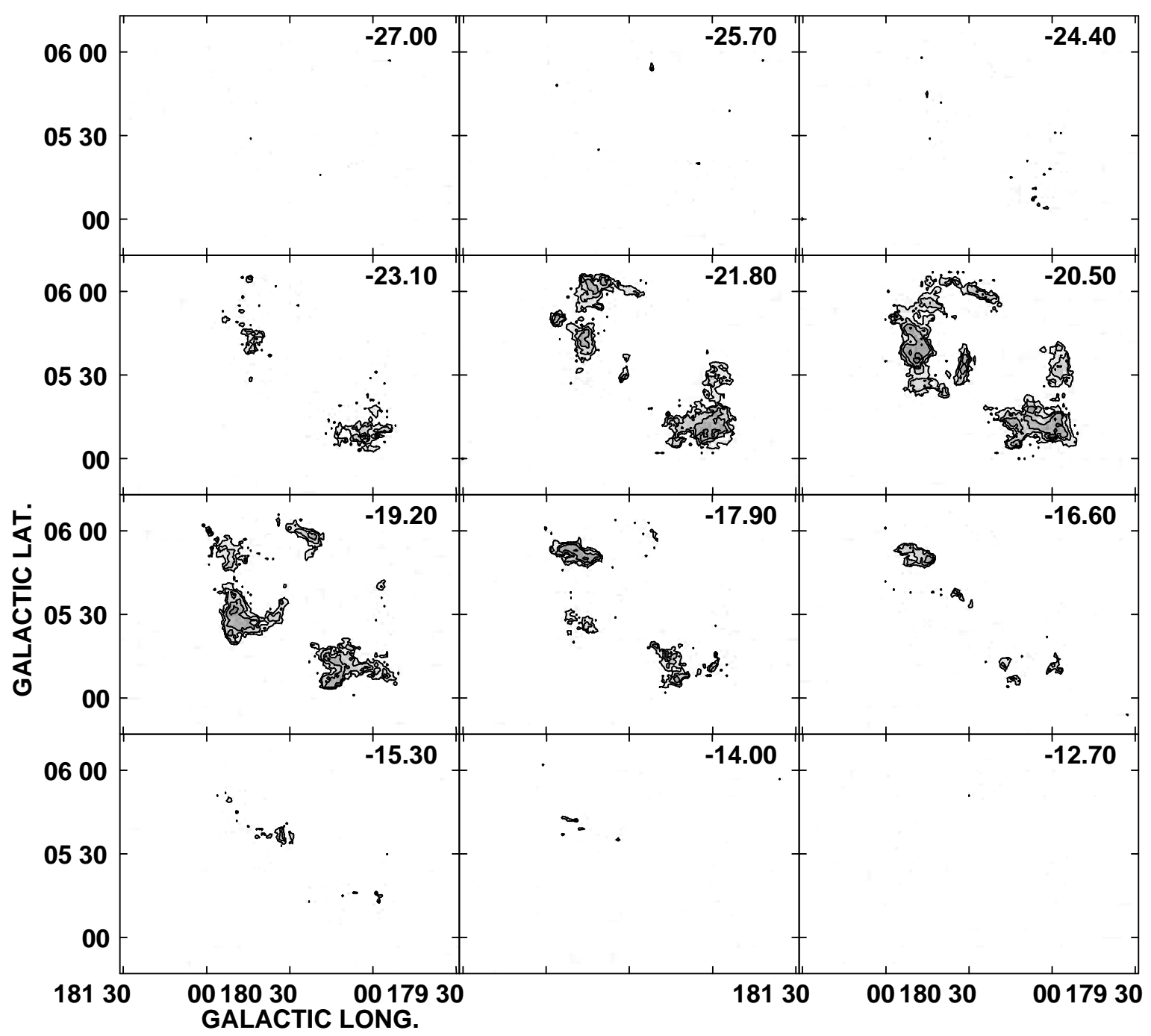

Figure 7. The ${ }^{12} \mathrm{CO}$ chanel map. For each channel map, two velocity channels of the spectrometer are binned together, thus velocity step is $1.3 \mathrm{~km} \mathrm{~s}^{-1}$. The grey scale range is from 0.4 to $8 \mathrm{~K} \mathrm{~km} \mathrm{~s}^{-1}$ and the lowest contour is $1 \mathrm{~K} \mathrm{~km} \mathrm{~s}^{-1}$, and the increment $2 \mathrm{~K} \mathrm{~km} \mathrm{~s}^{-1}$.

ity to be $\sim 1,100 \mathrm{~K} \mathrm{~km} \mathrm{~s}^{-1} \mathrm{pc}^{2}$, and ${ }^{13} \mathrm{CO}(J=1-0)$ luminosity, $110 \mathrm{~K} \mathrm{~km} \mathrm{~s}^{-1} \mathrm{pc}^{2}$ (see Table 1 ).

There are several ways to determine the linewidth of a cloud, and these have been discussed well in other papers (Lee 1994; Carpenter et al. 1993). We decompose the gas motions within each molecular cloud into two terms, prior to deriving the linewidths. These two independent motions have been discussed in Dickman \& Kleiner (1985), and in Lee et al. (1990). They represent two measurable motions within clouds: the first is an 'internal velocity dispersion' $\left(\sigma_{\mathfrak{i}}^{\mathrm{j}}\right)$, which represents the spread of velocities observed along the $\mathrm{j}$-th line of sight (LOS); and the second is a 'centroid velocity dispersion' $\left(\sigma_{c}\right)$, which is associated with the point-to-point bulk motions of the gas appearing in the cloud map - the square of this centroid velocity dispersion is simply defined to be the ensemble variance of the observed LOS centroid (i.e., LOS average) velocities.

Thus, the total velocity dispersion of a cloud can be represented as $\sigma_{t o t}=\sqrt{\sigma_{i}^{2}+\sigma_{c}^{2}}$. The cloud linewidth (FWHM) can be determined from: $\Delta V=\sqrt{8 \ln 2} \sigma_{t o t}$ assuming a Gaussian profile. We used ${ }^{12} \mathrm{CO}$ data for estimating linewidths in Table 1 . If we assume that a cloud has a spherical shape with a uniform density distribution, and that the turbulence is the main agent supporting the cloud against self-gravity, then we may estimate its virial mass using the equation:

$$
M_{V I R}=582 D \sigma_{\text {tot }}^{2} \quad\left[M_{\odot}\right] .
$$

where $D$ is the diameter of a cloud assuming a spherical cloud in units of pc. The viral mass can also be obtained from the linewidth of the entire cloud (MacLaren et al. 1988) as:

$$
M_{V I R}=190 r \Delta V^{2} \quad\left[M_{\odot}\right],
$$

where $r$ is radius of the cloud assuming a spherical shape in units of pc, and $\Delta V$ is the linewidth of the 


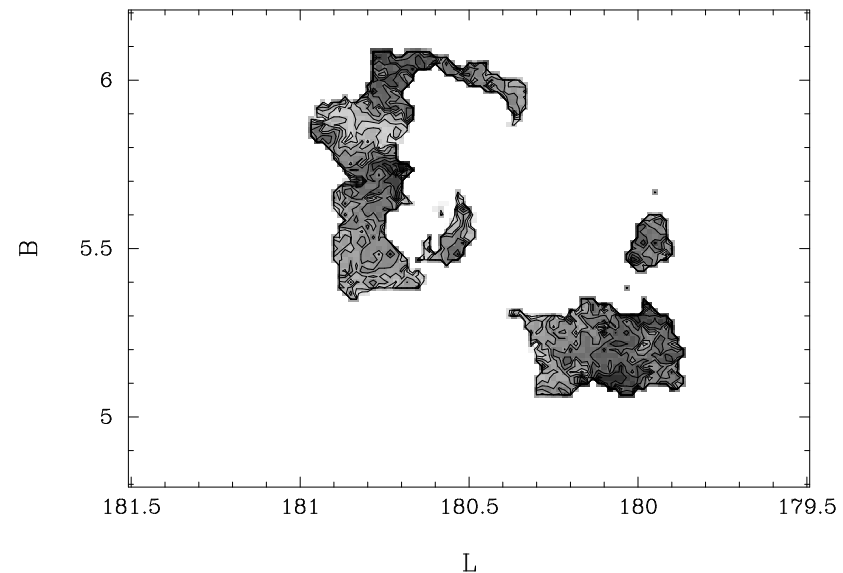

Figure 8. Mean velocity map above a threshold larger than $3 \sigma \mathrm{CO}$ integrated intensity. Contour level is starting from $2.8 \mathrm{~km} \mathrm{~s}^{-1}$ and its increment is $0.1 \mathrm{~km} \mathrm{~s}^{-1}$, and grey scale is ranging from 0 to $5 \mathrm{~km} \mathrm{~s}^{-1}$.

whole cloud in units of $\mathrm{km} \mathrm{s}^{-1}$. If, instead, we assume that each cloud has a spherical $r^{-1}$ density distribution, the corresponding virial mass will be $25 \%$ larger than that given by equation 1 (Lee 1994; Lee \& Jung 2003). We use equation 2 for estimating virial masses of the clouds. One general method for estimating cloud masses is to use the relationship between the CO integrated intensity and the molecular hydrogen column density, or equivalently that between $\mathrm{CO}$ luminosity $\left(L_{\mathrm{CO}}\right)$ and gas mass $\left(M_{\mathrm{CO}}\right)$. In fact, this method is favorable when a cloud is not gravitationally bound and thus virial mass estimate is not plausible, and LTE assumption is not applicable. The proportionality constant $X$, a conversion factor from CO luminosity (or integrated intensity) is used to estimate gas mass (or column density); it is implicitly assumed that such a factor has general applicability. We have used an estimate of this conversion factor: $X=2.3 \times 10^{20}$ $\mathrm{cm}^{-2}\left(\mathrm{~K} \mathrm{~km} \mathrm{~s}^{-1}\right)^{-1}$ (Bloemen 1989) - this value was established through a $\gamma$-ray analysis. The fact that ${ }^{13} \mathrm{CO}$ emission is much weaker than expected in some portions of the clouds drive us to use only ${ }^{12} \mathrm{CO}$ data for estimating physical parameters of the clouds. The derived physical parameters of the two clouds are tabulated in Table 1.

\section{Some Highlights AND Discussion}

Below we present some observational highlights and related discussion.

1. Our target clouds reside in the interarm region between the Orion and Perseus Arms since their distances are about $1.1 \mathrm{kpc}$ (Kim et al. 2000). To the Galactic anticenter direction, there is GEM OB1 association, the distance of which is $2 \mathrm{kpc}$ (Carpenter et al. 1994), at the exact center of Perseus Arm. Instellar environment of the clouds in the interarm region could be quite different; weaker interstellar field, less abundance etc. As seen from Figures 5 and 6 , the CO emission of the target clouds is confined to the velocity range from

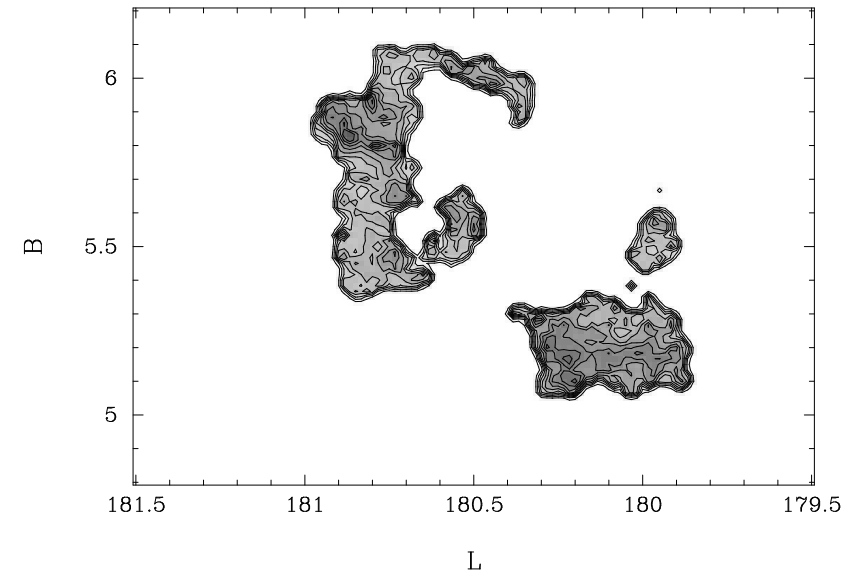

Figure 9. Linewidth map above a threshold larger than $3 \sigma$ CO integrated intensity. Contour level is starting from 1.6 $\mathrm{km} \mathrm{s}^{-1}$ and its increment is $0.12 \mathrm{~km} \mathrm{~s}^{-1}$, and grey scale is ranging from 1.5 to $4 \mathrm{~km} \mathrm{~s}^{-1}$.

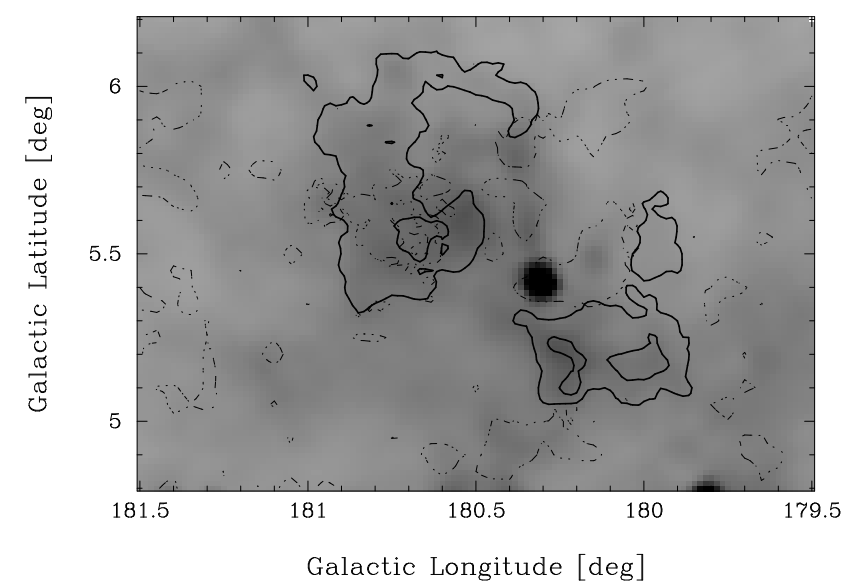

Figure 10. The IRAS 100 micron image and the $3 \sigma$ and $10 \sigma$ contours of ${ }^{12} \mathrm{CO}$ integrated intensity(in solid line) of the anomalous clouds. Overlayed dotted line is for the other clouds in the velocity range of -10 to $+10 \mathrm{~km} \mathrm{~s}^{-1}$.

-25 to $-15 \mathrm{~km} \mathrm{~s}^{-1}$. This implies that these clouds are running away from the Orion Arm towards the Perseus Arm, and located exactly in the middle of two arms. So far no other molecular clouds have been reported in this region. Gas clouds detached from the spiral arm may be caused by some ejection mechanism, such as supernova. Recently, Matsunaga et al. (2001) reported several molecular supershells in their NANTEN survey of the Galactic Plane. In fact, explosive phenomena could occur at any part of the spiral arms, and leave imprints of their activities. To clarify this phenomenon in the Galactic anticenter region, a more systematic survey with higher sensitivity for large area is required.

2. Comparing with other velocity components of the mapped region $\left(-10\right.$ to $0 \mathrm{~km} \mathrm{~s}^{-1}$ and 0 to $\left.10 \mathrm{~km} \mathrm{~s}^{-1}\right)$, the linewidths of the target clouds are broader $(\Delta V=$ 3.6 to $3.9 \mathrm{~km} \mathrm{~s}^{-1}$; see Table 1$)$. Linewidths of the other velocity ranges are 1 to $2.5 \mathrm{~km} \mathrm{~s}^{-1}$. In fact, they are somewhat broader than those of the normal dark clouds in the solar neighborhood without any in- 
Table 1

Physical parameters of the two clouds A and B

\begin{tabular}{cccccccccccc}
\hline & $l$ & $\delta l$ & $b$ & $\delta b$ & $\left\langle V_{L S R}\right\rangle$ & $\Delta V_{L S R}^{a}$ & $T_{p k}$ & size & $L_{C O}$ & $M_{V I R}^{b}$ & $M_{C O}$ \\
\hline & {$[\mathrm{deg}]$} & {$[\mathrm{deg}]$} & {$[\mathrm{deg}]$} & {$[\mathrm{deg}]$} & {$\left[\mathrm{km} \mathrm{s}^{-1}\right]$} & {$\left[\mathrm{km} \mathrm{s}^{-1}\right]$} & {$[\mathrm{K}]$} & {$[\mathrm{pc}]$} & {$\left[\mathrm{unit}^{c}\right]$} & $10^{3} M_{\odot}$ & $10^{3} M_{\odot}$ \\
\hline A & 180.10 & 0.26 & 5.20 & 0.16 & -20.5 & 3.9 & 5.4 & 8.6 & 530 & 12.4 & 1.9 \\
B & 180.81 & 0.12 & 5.64 & 0.40 & -20.0 & 3.6 & 3.7 & 10.8 & 570 & 13.3 & 2.1 \\
\hline
\end{tabular}

a Linewidth is estimated using ${ }^{12} \mathrm{CO}$

b Virial mass is estimated using Equation 2

${ }^{c}$ Unit is $\left[\mathrm{K} \mathrm{km} \mathrm{s}^{-1} \mathrm{pc}^{2}\right]$

ternal heating source $\left(\Delta V=1 \sim 2 \mathrm{~km} \mathrm{~s}^{-1}\right)$. This fact implies that there may have been some ejecting and disrupting mechanism, as mentioned above, which gave rise to negative LOS velocity of $-20 \mathrm{~km} \mathrm{~s}^{-1}$ and broader linewidth.

3. The virial mass estimate of two target clouds are much larger than the masses from CO luminosity. Because of the broader linewidths, their virial masses are sustantially overestimated. Thus, we use CO luminosity to estimate their masses, which are 1,900 and 2,100 solar masses (see Table 1). The mass estimate of the target clouds indicates that the cloud masses are near the upper limit of those of the dark clouds in the solar neighborhood. The weaker emission feature of ${ }^{13} \mathrm{CO}$ of the target clouds remaines fto be studied further with more sensitive observations.

4. As seen from Figure 8, there seems to be no systematic variation of the velocity field within the clouds, except a small area in Cloud B. Channel map(Figure 7) also shows that there is no significant change in their morphology. These facts indicate that there is no systematic motion within the clouds. The two clouds show only a systematic receding motion, away from the Orion Arm.

5. From Figure 9 of Paper I, the distribution of H I emission of the Weaver and Williams (1973) database is found to be in a very small dynamic range within a factor of 1.3. We have also checked the more recent H I data from Hartmann et al. (1997), and found no significant change. Thus the atomic hydrogen contribution to the FIR emission will not be significant when compared with the $\mathrm{CO}$ emission and FIR dust emission. Hence we did not include the $\mathrm{H}$ I contribution. The CO emission is, in general, well matched with the FIR emission region at $100 \mu \mathrm{m}$, when there is less foreground and background stray dust emission, thus the dust emission is all correlated with molecular clouds associated. As the target clouds are located just above the Galacic Plane, the foreground and backgrond dust emission are stirred in regular fashion except for the one IRAS point source (Figure 10). Thus FIR emission and the ${ }^{12} \mathrm{CO}$ integrated intensity relationship could not be established in directions toward the target clouds, except that highest-possible value of $I_{100} / I_{C O}$ is $\sim 0.38$ $\left(\mathrm{K} \mathrm{km} \mathrm{s}^{-1}\right) /\left(\right.$ MJy $s r^{-1}$ ) after subtracting minimum background emission. This value is very close to those of normal dark clouds (Lee \& Lee 2005).

\section{SUMMARY}

We have observed around $1 \mathrm{deg}^{2}$ section of $(l, b)=$ $\left(180^{\circ} .0-181^{\circ} .0,5^{\circ} .0-6^{\circ} .0\right)$ in the $(J=1-0)$ transitions of ${ }^{12} \mathrm{CO}$ and ${ }^{13} \mathrm{CO}$ using the 3 -mm SIS mixer receiver on the Taeduk Radio Astronomy Observatory (TRAO) on a $1^{\prime}$-grid. The mapped region covers the enire extent of the two clouds with anomalous velocities, which were identified in the previous Galactic anticenter CO survey (Paper I). The ${ }^{13} \mathrm{CO}(J=1-0)$ observations were focused only on the strong emission region of ${ }^{12} \mathrm{CO}(J=1-0)$. The results of the observations are presented in the form of integrated intensity maps, position-velocity maps, and channel maps. The integrated intensity map shows that the target clouds are well isolated from other clouds. We identified two sub-clouds, named A and B, and derived their physical parameters separately. These two clouds have similar characteristics. The radial velocity of the mapped region is found to fall in three velocity ranges of $V_{\mathrm{LSR}}=$ -25 to $-15 \mathrm{~km} \mathrm{~s}^{-1},-10$ to $0 \mathrm{~km} \mathrm{~s}^{-1}$ and 0 to 10 $\mathrm{km} \mathrm{s}^{-1}$. The position-velocity maps shows that there is no systematic change in the centroid velocity and mean velocity widths within the clouds. In an overall sense, the characteristic of the $\mathrm{CO}$ emissions from the clouds does not show any significant differences from those of dark clouds in the solar neighborhood in terms of basic physical parameters, such as temperature, mass and morphology. However, broader linewidth compared with those of solar neighborhood dark clouds, and location in the interarm region makes them distinguished objects. Further study toward a larger area including these clouds may clarify the possible ejection mechanism in more detail.

\section{ACKNOWLEDGMENTS}

Y. Lee is grateful for the continuing support from Korea Astronomy and Space Science Institute and Taeduk Radio Astronomy Observatory.

\section{REFERENCES}

Blitz, L. 1991. The Interstellar Disk-Halo Connection in Galaxies, ed. H. Bloeman, Kluwer, IAU Symp., 144, 41

Bloemen, J. B. G. M. 1989. Diffuse Galactic Gamma-Ray Emission, ARAA, 27, 469

Carpenter, J. M., Snell, R. L., \& Schloerb, F. P. 1993, Molecular Clouds Associated with Luminous FIR Sources in the Outer Galaxy, ApJ, 450, 201 
Carpenter, J. M., Snell, R. L., \& Schloerb, F. P. 1995, Anatomy of the Gemini OB1 Molecular Cloud Complex, ApJ, 445, 246

Dickman, R. L., \& Kleiner, S. C. 1985, Largescale Structure of the Taurus Molecular Complex - Part Three - Methods for Turbulence, ApJ, 295, 479

Hartmann, D., \& Burton, W. B. 1997, Atlas of Galactic Neutral Hydrogen (Cambridge: Cambridge University Press)

Kim, H.-G., Lee, Y., Park, B.-G., \& Kim, G.-G. 2000, Distance Determination to the Molecular Clouds in the Galactic Anti-Center Region, JKAS, 33, 151

Kutner, M. C., \& Ulich, B. L. 1981, Recommendations for Calibration of Millimeter-Wavelength Spectral Line Data, ApJ, 250, 341

Lee, Y., Snell, R. L., \& Dickman, R. L. 1990, Analysis of (C12) O and (C-13)O Emission in a 3 Square Degree Region of the Galactic Plane between $\mathrm{L}=23 \mathrm{deg}$ and $25 \mathrm{deg}$, ApJ, 355, 536

Lee, Y. 1994, Mass Estimate Techniques of Molecular
Clouds, Publ. Korean Astron. Soc., 9, 1, 55

Lee, Y., Jung, J. H., Chung, H. S., Kim, H.-G., Park, Y.S., Kim, H. R., Kim, B.-G., Kim, J., \& Han, S.-T. 1999, Galactic Anticenter CO Survey. I. Area $l=178(\mathrm{deg})$ to 186 (deg), $b=3.5$ to 6 (deg), A\&AS, 138, 187

Lee, Y., \& Jung, J. H. 2003, A Molecular Cloud Complex above the Galactic Plane. I. Extended CO Observations of the NGC 281 Region, New Astron., 8, 3, 191

Lee, Y., \& Lee, C. W. 2005 A Study of CO and FIR Dust Emission of Lynds 694/695 Dar Clouds, New Astron., 10, 463

MacLaren, I., \& Richardson, K. M. 1988, Corrections to Virial Estimates of the Molecular Cloud Masses, ApJ, 333,821

Weaver, H. F., \& Williams, D. R. W. 1973, A Berkeley Low-Latitude Survey of Neutral Hydrogen Part I. Profiles, A\&AS, 8, 1

Wouterloot, J. G. A., \& Brand, J. 1989, IRAS Sources beyond the Solar Circle. I - CO Observations, A\&AS, 80, 149 